\title{
A t-Norm Fuzzy Logic for Approximate Reasoning
}

\author{
Alex Tserkovny \\ Dassault Systemes, Boston, USA \\ Email: atserkovny@yahoo.com
}

How to cite this paper: Tserkovny, A. (2017) A t-Norm Fuzzy Logic for Approximate Reasoning. Journal of Software Engineering and Applications, 10, 639-662. https://doi.org/10.4236/jsea.2017.107035

Received: March 6, 2017

Accepted: June 26, 2017

Published: June 29, 2017

Copyright $\odot 2017$ by author and Scientific Research Publishing Inc. This work is licensed under the Creative Commons Attribution International License (CC BY 4.0).

http://creativecommons.org/licenses/by/4.0/

\begin{abstract}
A t-norm fuzzy logic is presented, in which a triangular norm (t-norm) plays the role of a graduated conjunction operator. Based on this fuzzy logic we develop methods for fuzzy reasoning in which antecedents and consequents involve fuzzy conditional propositions of the form "If $X$ is $A$ then $y$ is $B$ ", with $A$ and $B$ being fuzzy concepts (fuzzy sets). In this study, we present a systemic approach toward fuzzy logic formalization for approximate reasoning. We examine statistical characteristics of the proposed fuzzy logic. As the matter of practical interest, we construct a set of fuzzy conditional inference rules on the basis of the proposed fuzzy logic. Important features of these rules are investigated.
\end{abstract}

\section{Keywords}

Fuzzy Logic, t-Norm, Implication, Antecedent, Consequent, Modus-Ponens, Fuzzy Conditional Inference Rule

\section{Introduction}

In our daily life we often make inferences whose antecedents and consequents contain fuzzy concepts. Such an inference cannot be made adequately by the methods which are based either on classical two valued logic or on many valued logic. In order to make such an inference, Zadeh suggested an inference rule called "compositional rule of inference". Using this inference rule, he, Mamdani, Mizumoto et al., R. Aliev and A. Tserkovny suggested several methods for fuzzy reasoning in which the antecedent contain a conditional proposition with fuzzy concepts:

Ant 1: If $x$ is $P$ then $y$ is $Q$

Ant 2: $x$ is $P^{\prime}$ 
Cons: $y$ is $Q^{\prime}$.

Those methods are based on an implication operator in various fuzzy logics. This matter has been under discussion for the last couple decades [1]-[46].

In (1.1) $x$ and $y$ are the names of objects, and $P, P^{\prime}, Q$ and $Q^{\prime}$ are fuzzy concepts represented by fuzzy sets in universe of discourse $U, U, V$ and $V$, respectively. This form of inference may be viewed as a generalized modus ponens which reduces to modus ponens when $P^{\prime}=P$ and $Q^{\prime}=Q$. Let $P$ and $Q$ be fuzzy sets in $U$ and $V$ respectively and correspondent fuzzy sets be represented as such $P \subset U\left|\mu_{P}: U \rightarrow[0,1], Q \subset V\right| \mu_{Q}: V \rightarrow[0,1]$, where

$$
P=\int_{U} \mu_{P}(u) / u, \quad Q=\int_{V} \mu_{Q}(v) / v .
$$

And let $\times, \cup, \cap, \neg$ and $\oplus$ be Cartesian product, union, intersection, complement and bounded-sum for fuzzy sets, respectively. Then the following fuzzy relations in $U \times V$ can be derived from fuzzy conditional proposition "If $x$ is $P$ then $y$ is $Q$ " in Ant 1 of (1.1). The fuzzy relations $R_{m}$ and $R_{a}$ were proposed by Zadeh [42] [43] [44] [45], $R_{c}$ by Mamdani [29], $R_{s}, R_{g}$ are by Mizumoto [32] [33], $R_{L 1}, R_{L 2}, R_{L 3}$ and $R_{L 4}$ are by Aliev and Tserkovny [2] [3] [4] [5], which are

$$
R_{L 1}=(P \times V \underset{L 1}{\Rightarrow} U \times Q)=\int_{U \times V}\left[\mu_{P}(u) \underset{L 1}{\rightarrow} \mu_{Q}(v)\right] /(u, v)
$$

where

$$
\begin{gathered}
\mu_{P}(u) \underset{L 1}{\rightarrow} \mu_{Q}(v)= \begin{cases}1-\mu_{P}(u), & \mu_{P}(u)<\mu_{Q}(v), \\
1, & \mu_{P}(u)=\mu_{Q}(v), \\
\mu_{Q}(v), & \mu_{P}(u)>\mu_{Q}(v) .\end{cases} \\
R_{L 2}=(P \times V \underset{L 2}{\Rightarrow} U \times Q)=\int_{U \times V}\left[\mu_{P}(u) \underset{L 2}{\rightarrow} \mu_{Q}(v)\right] /(u, v)
\end{gathered}
$$

where

$$
\begin{gathered}
\mu_{P}(u) \underset{L 2}{\rightarrow} \mu_{Q}(v)= \begin{cases}1, & \mu_{P}(u) \leq \mu_{Q}(v), \\
\left(1-\mu_{P}(u)\right) \wedge \mu_{Q}(v), & \mu_{P}(u)>\mu_{Q}(v) .\end{cases} \\
R_{L 3}=(P \times V \underset{L 3}{\Rightarrow} U \times Q)=\int_{U \times V}\left[\mu_{P}(u) \underset{L 3}{\rightarrow} \mu_{Q}(v)\right] /(u, v)
\end{gathered}
$$

where

$$
\begin{gathered}
\mu_{P}(u) \underset{L 3}{\rightarrow} \mu_{Q}(v)= \begin{cases}1, & \mu_{P}(u) \leq \mu_{Q}(v), \\
\frac{\mu_{Q}(v)}{\mu_{P}(u)+\left(1-\mu_{Q}(v)\right)}, & \mu_{P}(u)>\mu_{Q}(v) .\end{cases} \\
R_{L 4}=(P \times V \underset{L 4}{\Rightarrow} U \times Q)=\int_{U \times V}\left[\mu_{P}(u) \underset{L 4}{\rightarrow} \mu_{Q}(v)\right] /(u, v)
\end{gathered}
$$

where

$$
\mu_{P}(u) \underset{L 4}{\rightarrow} \mu_{Q}(v)= \begin{cases}\frac{1-\mu_{P}(u)+\mu_{Q}(v)}{2}, & \mu_{P}(u)>\mu_{Q}(v), \\ 1, & \mu_{P}(u) \leq \mu_{Q}(v) .\end{cases}
$$


A necessary consideration for this discussion is that with the only few exceptions for S-logic (1.6) and G-logic (1.7), and L1-L4(1.3)-(1.6) all other known fuzzy logics don't satisfy either the classical "modus-ponens" principal, or other criteria which fit human intuition and first formulated in [32]. The proposed fuzzy logic has an implication operator, which satisfies the "modus-ponens" principal and criteria, which fit human intuition.

The second section of the article will cover some initial fuzzy logic creation considerations. In third section a set of operations in proposed fuzzy logic is presented. The fourth section is devoted to an introduction of a t-norm as a graduated conjunction operator in presented fuzzy logic. The Section five will cover a power sets based features of proposed fuzzy logic. The statistical analysis of the fuzzy logic is completed in Section six. Section Seven covers the issue of fuzzy conditional inference rules based on proposed fuzzy logic and extended investigation of its features.

\section{Preliminary Considerations}

In order to start formulating of a fuzzy logic major implication operator, we are proposing the following function as a part of it:

$$
F_{1}(p, q)=\neg p \cdot q=(1-p) \cdot q
$$

\section{Definition 1.}

An implication function is a continuous function $I$ from $[0,1] \times[0,1]$ into $[0,1]$ such that the following properties hold for $\forall p, p^{\prime}, q, q^{\prime}, r \in[0,1]$ :

(I1) If $p \leq p^{\prime}$ then $I(p, q) \geq I\left(p^{\prime}, q\right)$;

(I2) If $q \leq q^{\prime}$ then $I(p, q) \leq I\left(p, q^{\prime}\right)$;

(I3) $I(0, q)=1$, (Falsity Principle);

(I4) $I(1, q) \leq q$, (Neutrality Principle);

(I5) $I(p, I(q, r))=I(q, I(p, r))$, (Exchange Principle);

(I6) $I(p, q)=I(n(q), n(p))$. (Contra positive Symmetry Principle), where $n($.$) -is a negation, which could be defined for n(q)$ as $T(\neg Q)=1-T(Q)$.

Before proving that $I(p, q)$ defined as

$$
I(p, q)=\left\{\begin{array}{l}
F_{1}(p, q), p>q ; \\
1, p \leq q
\end{array}\right.
$$

and $F_{1}(p, q)$ is from (2.1) satisfies (I1)-(I6) axioms, let us show some basic operations in proposed fuzzy logic.

\section{The Fuzzy Logic}

Let us designate the truth values of logical antecedent $P$ and consequent $Q$ as $T(P)=p$ and $T(Q)=q$ respectively. Then relevant set of proposed fuzzy logic operators are shown in Table 1.

In other words we propose a new many-valued system, characterized by the set of base union $(\cup)$ and intersection $(\bigcap)$ operations with relevant complement, defined as $T(\neg P)=1-T(P)$. In addition, the operators $\downarrow$ and $\uparrow$ are 
Table 1. Relevant set of proposed fuzzy logic operators.

\begin{tabular}{|c|c|c|}
\hline Name & Designation & Value \\
\hline Tautology & $P^{\mathrm{I}}$ & 1 \\
\hline Controversy & $P^{\mathrm{O}}$ & 0 \\
\hline Negation & $\neg P$ & $1-P$ \\
\hline Disjunction & $P \vee Q$ & $\left\{\begin{array}{l}p \cdot q, p+q<1 \\
1, p+q \geq 1\end{array}\right.$ \\
\hline Conjunction & $P \wedge Q$ & $\left\{\begin{array}{l}p \cdot q, p+q>1 \\
0, p+q \leq 1\end{array}\right.$ \\
\hline Implication & $P \rightarrow Q$ & $\left\{\begin{array}{l}(1-p) \cdot q, p>q \\
1, p \leq q\end{array}\right.$ \\
\hline Equivalence & $P \leftrightarrow Q$ & $\left\{\begin{array}{l}(1-p) \cdot q, p<q \\
1, p=q \\
(1-q) \cdot p, q<p\end{array}\right.$ \\
\hline Pierce Arrow & $P \downarrow Q$ & $\left\{\begin{array}{l}(1-p) \cdot(1-q), p+q<1 \\
0, p+q \geq 1\end{array}\right.$ \\
\hline Shaffer Stroke & $P \uparrow Q$ & $\left\{\begin{array}{l}(1-p) \cdot(1-q), p+q>1, \\
1, p+q \leq 1\end{array}\right.$ \\
\hline
\end{tabular}

expressed as negations of the $U$ and $\cap$ correspondingly. It is a well-known fact that the operation implication in a fuzzy logic was the foundation of decision making procedure for numerous approximate reasoning tasks. Therefore let us prove that proposed implication operation from (2.2) satisfies axioms (I1)(I6). For this matter let us pose the problem very explicitly. We are working in many-valued system, which for present purposes is all or some of the real interval $\mathfrak{R}=[0,1]$. As was mentioned in [1], the rationales there are more than ample for our purposes in very much of practice, the following set $\{0,0.1,0.2, \cdots, 0.9,1\}$ of 11 values is quite sufficient, and we shall use this set $V_{11}$ in our illustration. Table 2 shows the operation implication in proposed fuzzy logic.

Theorem 1. Let a continuous function $I(p, q)$ is defined in (2.3) and its values are from a Table 2, i.e.

$$
I(p, q)=\left\{\begin{array}{l}
F_{1}(p, q), p>q ; \\
1, p \leq q,
\end{array}=\left\{\begin{array}{l}
(1-p) \cdot q, p>q, \\
1, p \leq q
\end{array}\right.\right.
$$

Where function $F(p, q)^{\text {norm }}$ is defined in (1), then axioms (I1)-(I6) are satisfied and, therefore it is an implication operation.

Where function $F(p, q)^{\text {norm }}$ is defined in (1), then axioms (I1)-(I6) are satisfied and, therefore it is an implication operation.

Proof:

$$
\text { (I1): } \begin{aligned}
& \forall p, p^{\prime} \in[0,1] \mid p^{\prime} \geq p>q \\
& \Rightarrow I(p, q)-I\left(p^{\prime}, q\right)=(1-p) \cdot q-\left(1-p^{\prime}\right) \cdot q=\left(p^{\prime}-p\right) \cdot q \mid p^{\prime}-p \geq 0
\end{aligned}
$$


Table 2. The operation implication in proposed fuzzy logic.

\begin{tabular}{cccccccccccc}
\hline$p \rightarrow q$ & $\mathbf{0}$ & $\mathbf{0 . 1}$ & $\mathbf{0 . 2}$ & $\mathbf{0 . 3}$ & $\mathbf{0 . 4}$ & $\mathbf{0 . 5}$ & $\mathbf{0 . 6}$ & $\mathbf{0 . 7}$ & $\mathbf{0 . 8}$ & $\mathbf{0 . 9}$ & $\mathbf{1}$ \\
\hline $\mathbf{0}$ & 1 & 1 & 1 & 1 & 1 & 1 & 1 & 1 & 1 & 1 & 1 \\
$\mathbf{0 . 1}$ & 0 & 1 & 1 & 1 & 1 & 1 & 1 & 1 & 1 & 1 & 1 \\
$\mathbf{0 . 2}$ & 0 & 0.08 & 1 & 1 & 1 & 1 & 1 & 1 & 1 & 1 & 1 \\
$\mathbf{0 . 3}$ & 0 & 0.07 & 0.14 & 1 & 1 & 1 & 1 & 1 & 1 & 1 & 1 \\
$\mathbf{0 . 4}$ & 0 & 0.06 & 0.12 & 0.18 & 1 & 1 & 1 & 1 & 1 & 1 & 1 \\
$\mathbf{0 . 5}$ & 0 & 0.05 & 0.1 & 0.15 & 0.2 & 1 & 1 & 1 & 1 & 1 & 1 \\
$\mathbf{0 . 6}$ & 0 & 0.04 & 0.08 & 0.12 & 0.16 & 0.2 & 1 & 1 & 1 & 1 & 1 \\
$\mathbf{0 . 7}$ & 0 & 0.03 & 0.06 & 0.09 & 0.12 & 0.15 & 0.18 & 1 & 1 & 1 & 1 \\
$\mathbf{0 . 8}$ & 0 & 0.02 & 0.04 & 0.06 & 0.08 & 0.1 & 0.12 & 0.14 & 1 & 1 & 1 \\
$\mathbf{0 . 9}$ & 0 & 0.01 & 0.02 & 0.03 & 0.04 & 0.05 & 0.06 & 0.07 & 0.08 & 1 & 1 \\
$\mathbf{1}$ & 0 & 0 & 0 & 0 & 0 & 0 & 0 & 0 & 0 & 0 & 1 \\
\hline
\end{tabular}

$$
\Rightarrow I(p, q) \geq I\left(p^{\prime}, q\right)
$$

whereas

$$
q \geq p^{\prime} \geq p \Rightarrow I(p, q)-I\left(p^{\prime}, q\right) \equiv 0 \Rightarrow I(p, q) \equiv I\left(p^{\prime}, q\right)
$$

(I2): $\forall q, q^{\prime} \in[0,1] \mid q \leq q^{\prime}<p$

$$
\begin{gathered}
\Rightarrow I(p, q)-I\left(p, q^{\prime}\right)=(1-p) \cdot q-(1-p) \cdot q^{\prime}=(1-p) \cdot\left(q-q^{\prime}\right) \mid q-q^{\prime} \leq 0 \\
\Rightarrow I(p, q) \leq I\left(p, q^{\prime}\right)
\end{gathered}
$$

whereas

$$
p \leq q \leq q^{\prime} \Rightarrow I(p, q)-I\left(p, q^{\prime}\right) \equiv 0 \Rightarrow I(p, q) \equiv I\left(p, q^{\prime}\right)
$$

(I3): $I(0, q) \equiv 1, q \geq 0$;

(I4): $I(1, q) \equiv 0 \Rightarrow I(1, q) \leq q$;

(I5): Since $\forall p, q, r \in[0,1] \mid p>q>r \Rightarrow q \rightarrow r=(1-q) \cdot r$, then

$$
p \rightarrow(q \rightarrow r)=(1-p) \cdot(1-q) \cdot r
$$

Whereas

$$
p \rightarrow r=(1-p) \cdot r \Rightarrow q \rightarrow(p \rightarrow r)=(1-q) \cdot(1-p) \cdot r
$$

(I6):

$$
I(n(q), n(p))=(1-q) \rightarrow(1-p)=\left\{\begin{array}{l}
q \cdot(1-p), 1-q>p, \\
1,1-q \leq p
\end{array}=\left\{\begin{array}{l}
(1-p) \cdot q, p>q, \\
1, p \leq q .
\end{array}\right.\right.
$$

In addition proposed fuzzy logic is characterized by the following features:

Commutativity for both conjunction $(\cap)$ and disjunction $(U)$ operations, i.e.: $p \wedge q=q \wedge p$ and $p \vee q=q \vee p$;

Assotiativity for these operations:

$$
p \wedge(q \wedge r)=(p \wedge q) \wedge r
$$


and

$$
p \vee(q \vee r)=(p \vee q) \vee r
$$

Distributivity:

$$
\begin{aligned}
& (p \vee q) \wedge r=(p \wedge r) \vee(q \wedge r), \\
& p \vee(q \wedge r)=(p \vee q) \wedge(p \vee r) .
\end{aligned}
$$

To prove the feature (3.4) note that

$$
\begin{gathered}
(p \vee q) \wedge r=\left\{\begin{array}{l}
(p \cdot q) \wedge r, p+q<1, \\
1 \wedge r, p+q \geq 1
\end{array}\right. \\
(p \cdot q) \wedge r=\left\{\begin{array}{l}
p \cdot q \cdot r, p \cdot q+r>1, \\
0, p \cdot q+r \leq 1
\end{array}, 1 \wedge r=r\right.
\end{gathered}
$$

On the other hand

$$
q \wedge r=\left\{\begin{array}{l}
q \cdot r, q+r>1 \\
0, q+r \leq 1
\end{array}\right.
$$

To prove the Feature (3.5) by using (3.7) we have

$$
\begin{gathered}
p \vee(q \wedge r)=p \vee\left\{\begin{array}{l}
q \cdot r, q+r>1, \\
0, q+r \leq 1
\end{array}=\left\{\begin{array}{l}
p \cdot q \cdot r, p \cdot q+r>1, \\
0, p \cdot q+r \leq 1
\end{array}\right.\right. \\
(p \vee q) \wedge(p \vee r)=p \vee(q \wedge r)
\end{gathered}
$$

Therefore the Expression (3.8) equals (3.9) Q.E.D.

DeMorgan theorems, which are extrapolated over the $M=[0,1]$ :

$$
\begin{aligned}
& \neg(p \wedge q)=\neg p \vee \neg q, \\
& \neg(p \vee q)=\neg p \wedge \neg q ;
\end{aligned}
$$

To prove these theorems notice that

$$
\neg(p \wedge q)=\left\{\begin{array}{l}
\neg(p \cdot q), p+q>1, \\
1, p+q \leq 1
\end{array}\right.
$$

On the other hand

$$
\neg p \vee \neg q=\left\{\begin{array}{l}
\neg p \cdot \neg q, \neg p+\neg q<1, \\
1, \neg p+\neg q \geq 1
\end{array}=\left\{\begin{array}{l}
\neg(p \cdot q), p+q>1, \\
1, p+q \leq 1
\end{array}\right.\right.
$$

Therefore the Expression (3.10) equals (3.11) Q.E.D. By analogous

$$
\neg(p \vee q)=\left\{\begin{array}{l}
\neg(p \cdot q), p+q<1, \\
0, p+q \geq 1
\end{array}\right.
$$

On the other hand

$$
\neg p \wedge \neg q=\left\{\begin{array}{l}
\neg p \cdot \neg q, \neg p+\neg q>1, \\
0, \neg p+\neg q \leq 1
\end{array}\right.
$$

Therefore the Expression (3.12) equals (3.13) Q.E.D. It should be mentioned that proposed fuzzy logic could also be characterized by yet another featured $\neg p=p$. As a conclusion we should admit that all above features confirm that 
resulting system can be applied to $V_{I I}$ for every finite and infinite $n$ up to that $\left(V_{1 l}, \neg, \wedge, \vee, \rightarrow\right)$ is then closed under all its operations.

\section{The t-Norm}

\section{Proposition.}

In proposed fuzzy logic the operation of conjunction

$$
\operatorname{Conj}(p, q)=\left\{\begin{array}{l}
p \cdot q, p+q>1, \\
0, p+q \leq 1
\end{array}\right.
$$

is a t-norm.

\section{Proof:}

The function $\operatorname{Conj}(p, q)$ is a t-norm if the following is true

1) Commutativity: $p \wedge q=q \wedge p$

2) Associativity: $(p \wedge q) \wedge r=p \wedge(q \wedge r)$

3) Monotonity: $p \leq q, p \wedge r \leq q \wedge r$

4) Neutrality: $1 \wedge p=p$

5) Absorption $0 \wedge p=0$

Commutativity:

$$
\operatorname{Conj}(p, q)=P \cap Q=\left\{\begin{array}{l}
p \cdot q, p+q>1, \\
0, p+q \leq 1
\end{array}\right.
$$

and

$$
\operatorname{Conj}(q, p)=Q \cap P=\left\{\begin{array}{l}
q \cdot p, q+p>1 \\
0, q+p \leq 1
\end{array}\right.
$$

therefore

$$
\operatorname{Conj}(p, q)=\operatorname{Conj}(q, p)
$$

Associativity:

Case: $\operatorname{Conj}(p, q) \wedge r$

$$
\operatorname{Conj}(p, q)=p \cdot q, p+q>1
$$$$
\Rightarrow \operatorname{Conj}(p, q) \wedge r=\left\{\begin{array}{l}
\operatorname{Conj}(p, q) \cdot r, \operatorname{Conj}(p, q)+r>1 \\
0, \operatorname{Conj}(p, q)+r \leq 1
\end{array}=\left\{\begin{array}{l}
p \cdot q \cdot r, p+q+r>1 \\
0, p+q+r \leq 1
\end{array}\right.\right.
$$

From where we have that

$$
\operatorname{Conj}(p, r)=\left\{\begin{array}{l}
p \cdot q \cdot r, p+q+r>1 \\
0, p+q+r \leq 1
\end{array}\right.
$$

For the case $p \wedge \operatorname{Conj}(q, r)$, where

$$
\operatorname{Conj}(q, r)=Q \cap R=\left\{\begin{array}{l}
q \cdot r, q+r>1, \\
0, q+r \leq 1
\end{array}\right.
$$

Using (4.3) we are getting similar to (4.2) results

$$
\begin{aligned}
p \wedge \operatorname{Conj}(q, r) & =\left\{\begin{array}{l}
p \cdot q \cdot r, p+q+r>1 \\
0, p+q+r \leq 1
\end{array}\right. \\
\operatorname{Conj}(p, q) & =p \wedge \operatorname{Conj}(q, r)
\end{aligned}
$$


Monotonity:

If $p \leq q \Rightarrow p \wedge r \leq q \wedge r$ then given

$$
p \wedge r=\left\{\begin{array}{l}
p \cdot r, p+r>1, \\
0, p+r \leq 1
\end{array} \text { and } q \wedge r=\left\{\begin{array}{l}
q \cdot r, q+r>1 \\
0, q+r \leq 1
\end{array}\right.\right.
$$

we are getting the following

$$
p \cdot r \leq q \cdot r \mid p \leq q
$$

Neutrality:

$$
1 \wedge p=\left\{\begin{array}{l}
1 \cdot p, 1+p>1 \\
0,1+p \leq 1
\end{array}=1\right.
$$

Absorption:

$$
0 \wedge p=\left\{\begin{array}{l}
0 \cdot p, p>1 \\
0, p \leq 1
\end{array}=0\right.
$$

\section{Fuzzy Power Sets and the Fuzzy Logic}

Definition 2. [9]

Given a fuzzy implication operator $\rightarrow$ and a fuzzy subset $Q$ of a crisp universe $U$, the fuzzy power set $P Q$ of $Q$ is given by the membership function $\mu_{P Q}$, with

$$
\mu_{P Q}(x)=\bigcap_{x \in U}\left(\mu_{P} x \rightarrow \mu_{Q} x\right)
$$

The degree to which $P$ is subset of $Q$ is

$$
\pi(P \subseteq Q)=\mu_{P Q}(x)
$$

Definition 3. [9]

Where conditions are as in Definition 2, the degree to which the fuzzy sets $P$ and $Q$ is the same, or their degree of sameness, is

$$
\pi(P \equiv Q)=\pi(P \subseteq Q) \wedge \pi(P \supseteq Q)
$$

The following is then immediate.

Proposition 1. [9]

$$
\pi(P \equiv Q)=\bigcap_{x \in U}\left(\mu_{P} X \leftrightarrow \mu_{Q} X\right)
$$

Based on (5.1)-(5.3) and taken into account (3.1) we can formulate the following.

Proposition 2. [9] (Degree of possibility of set-inclusion)

$$
\pi(P \subseteq Q)=\left\{\begin{array}{l}
\left(1-\mu_{P} x\right) \cdot \mu_{Q} x, \mu_{P} x>\mu_{Q} X, \\
1, \mu_{P} X \leq \mu_{Q} X,
\end{array}\right.
$$

Proposition 3. (Degree of possibility of set-equality)

$$
\pi(P \equiv Q)=\left\{\begin{array}{l}
\left(1-\mu_{P} x\right) \cdot \mu_{Q} x, \mu_{P} x>\mu_{Q} x, \\
1, \mu_{P} x=\mu_{Q} x, \\
\left(1-\mu_{Q} x\right) \cdot \mu_{P} x, \mu_{Q} x<\mu_{P} x,
\end{array}\right.
$$


From (4-4) is clear that $\pi(P \equiv Q)=1$ in case when $\mu_{P} X=\mu_{Q} X$. As it was mentioned in [9] there seem to be two plausible ways to define the degree to which sets $P$ and $Q$ may be said to be disjointed. One is the degree to which each is a subset of the other's complement. The second is the degree to which their intersection is empty.

Definition 4. [9] The degree of disjointness of $P$ and $Q$, or degree to which $P$ and $Q$ are disjointed, in the first and second sense, are

(1) $\pi\left(\right.$ Pdisj $\left._{1} Q\right)=\pi\left(P \subseteq Q^{C}\right) \wedge \pi\left(Q \supseteq B^{C}\right)$

(2) $\pi\left(\operatorname{Pdisj}_{2} Q\right)=\pi(P \cap Q \equiv \varnothing)$.

For the case (1)

$$
\begin{aligned}
& \pi\left(P \subseteq Q^{C}\right)=\mu_{P} x \rightarrow\left(1-\mu_{Q} x\right)=\left\{\begin{array}{l}
\left(1-\mu_{P} x\right) \cdot\left(1-\mu_{Q} x\right), \mu_{P} x>\left(1-\mu_{Q} x\right), \\
1, \mu_{P} x \leq\left(1-\mu_{Q} x\right),
\end{array}\right. \\
& \pi\left(Q \supseteq P^{C}\right)=\mu_{Q} x \rightarrow\left(1-\mu_{P} x\right)=\left\{\begin{array}{l}
\left(1-\mu_{Q} x\right) \cdot\left(1-\mu_{P} x\right), \mu_{Q} x>\left(1-\mu_{P} x\right), \\
1, \mu_{Q} x \leq\left(1-\mu_{P} x\right),
\end{array}\right.
\end{aligned}
$$

Therefore from (5.5) and (5.6) definition (1) looks like

$$
\pi\left(\text { Pdisj }_{1} Q\right)=\left\{\begin{array}{l}
\left(1-\mu_{P} x\right) \cdot\left(1-\mu_{Q} x\right), \mu_{P} x+\mu_{Q} x>1, \\
1, \mu_{P} x+\mu_{Q} x \leq 1
\end{array}\right.
$$

For the case (2)

$$
\pi\left(\operatorname{Pdisj}_{2} Q\right)=\mu_{P} x \wedge \mu_{Q} X=\left\{\begin{array}{l}
\mu_{P} x \cdot \mu_{Q} X, \mu_{P} x+\mu_{Q} x>1, \\
0, \mu_{P} X+\mu_{Q} x \leq 1
\end{array}\right.
$$

Definition 5. [9] (Degree to which a set is a subset of its complement). The expression

$$
\pi\left(P \subseteq P^{C}\right)=\mu_{P} x \rightarrow\left(1-\mu_{P} x\right)=\left\{\begin{array}{l}
\left(1-\mu_{P} x\right)^{2}, \mu_{P} x>0.5, \\
1, \mu_{P} x \leq 0.5,
\end{array}\right.
$$

Definition 6. [9] (Degree to which a set is disjointed from its complement, in the two senses). From (5.7), (5.8) the following is taking place

The value of $\pi\left(\operatorname{Pdisj}_{1} P^{C}\right) \equiv 1$, whereas $\pi\left(\operatorname{Pdisj}_{2} P^{C}\right) \equiv 0$.

\section{Statistical Property of the Fuzzy Logic}

In this chapter we discuss some properties of proposed fuzzy implication operator (3.1), assuming that the two propositions (antecedent/consequent) in a given compound proposition are independent of each other and the truth values of the propositions are uniformly distributed [20] on the interval [0,1]. In other words we assume that the propositions $P$ and $Q$ are independent of each other and the truth values $v(P)$ and $v(Q)$ are uniformly distributed across the interval $[0,1]$. Let $p=v(P), q=v(Q)$. Then the value of the implication $I=v(p \rightarrow q)$ is some function $I=I(p, q)$.

Because $p$ and $q$ are assumed to be uniformly and independently distributed across $[0,1]$, the expected value of the implication is 


$$
E(I)=\iint_{\Re} I(p, q) \mathrm{d} p \mathrm{~d} q
$$

And its variance is

$$
\operatorname{Var}(I)=E\left[(I-E(I))^{2}\right]=\iint_{\mathfrak{R}}(I(p, q)-E(I))^{2} \mathrm{~d} p \mathrm{~d} q=E\left[I^{2}\right]-E[I]^{2}
$$

where $\mathfrak{R}=\{(p, q): 0 \leq p \leq 1,0 \leq q \leq 1\}$. From (6.1) and given Expression (3.1) and the fact that

$$
I(p, q)=\left\{\begin{array}{l}
I_{1}(p, q), p>q, \\
I_{2}(p, q), p \leq q
\end{array}\right.
$$

we have the following

But from Table 2 it is clear that for $I_{1}(p, q)$ we have

$$
\mathfrak{R}_{1}=\{(p, q): 0.2 \leq p \leq 0.9,0.1 \leq q \leq 0.8\}
$$

because of the following

$$
\begin{aligned}
& I_{1}(1, q) \equiv 0 \text { and } I_{1}(p, 0) \equiv 0, \text { therefore } \\
& \qquad E\left(I_{1}\right)=\iint_{\Re_{1}} I_{1}(p, q) \mathrm{d} p \mathrm{~d} q=\int_{0.1}^{0.80 .9} \int_{0.2}^{0.9}[(1-p) \cdot q] \mathrm{d} p \mathrm{~d} q=\int_{0.1}^{0.8} q \int_{0.2}^{0.9}(1-p) \mathrm{d} p \mathrm{~d} q .
\end{aligned}
$$

Given (6.3) the following is true

$$
\int_{0.2}^{0.9}(1-p) \mathrm{d} p=\left.\left(p-p^{2} \cdot 1 / 2\right)\right|_{p=0.2} ^{p=0.9}=0.325
$$

Whereas

$$
\int_{0.1}^{0.8} q \mathrm{~d} q=\left.\left(q^{2} \cdot 1 / 2\right)\right|_{p=0.1} ^{p=0.8}=0.325
$$

From (6.4) and (6.5) $E\left(I_{1}\right)=0.325^{2}=0.105625$. Whereas $E\left(I_{2}\right)=1$. Let us notice that for the most implications we have the following

$$
\frac{\operatorname{Card}\left(I_{1}\right)}{\operatorname{Card}\left(I_{2}\right)} \approx 0.8 \Rightarrow E(I)=0.55 \cdot E\left(I_{2}\right)+0.45 \cdot E\left(I_{1}\right)=0.598
$$

From (6.2) we have

$$
I_{1}^{2}(p, q)=[(1-p) \cdot q]^{2}=[q-p \cdot q]^{2}=q^{2}-2 p q^{2}+p^{2} q^{2}
$$

From (6.6) finally we have

$$
\begin{aligned}
& I_{1}^{2}(p, q)=q^{2} \cdot\left(1-2 p+p^{2}\right) \\
& \Rightarrow E\left(I_{1}^{2}\right)=\iint_{\Re_{1}} I_{1}^{2}(p, q) \mathrm{d} p \mathrm{~d} q=\left.\left.\frac{q^{3}}{3}\right|_{q=0.1} ^{q=0.8} \cdot\left(p-\frac{p^{2}}{2}+\frac{p^{3}}{3}\right)\right|_{p=0.2} ^{p=0.9}=0.056898 .
\end{aligned}
$$

Whereas $E\left(I_{2}^{2}\right)=1$.

Therefore $E\left(I^{2}\right)=\left(E\left(I_{1}^{2}\right)+E\left(I_{2}^{2}\right)\right) / 2=0.528449$ From (6.2) we have

$$
\operatorname{Var}(I)=0.170845
$$

Both values of $E(I)$ and $\operatorname{Var}(I)$ demonstrate that the proposed fuzzy implication operator could be considered as a second of the fuzziest implication 
from the list [34] of known so far. In addition to that feature it satisfies the set of important Criteria I-IV, which is not the case for the most above mentioned implication operators.

\section{The Fuzzy Logic and Fuzzy Conditional Inference}

As it was mentioned in [32] in the semantics of natural language there exist vast amounts of concepts and we humans very often make inferences antecedents and consequences of which contain fuzzy concepts. Therefore, from the standpoint of artificial intelligence, it seems that formalization of inference methods for such inferences is very important. Following a well-known pattern, established a couple of decades ago and the standard approaches toward such formalization, let $U$ and $V$ (from now on) be two universes of discourses and correspondent fuzzy sets be represented as such

$$
P \subset U\left|\mu_{P}: U \rightarrow[0,1], Q \subset V\right| \mu_{Q}: V \rightarrow[0,1]=0.170845
$$

where

$$
P=\int_{U} \mu_{P}(u) / u
$$

and

$$
Q=\int_{V} \mu_{Q}(v) / v
$$

Whereas given (7.1) and (7.2) a binary relationship for the fuzzy conditional proposition of the type: "If $X$ is $P$ then $y$ is $Q$ " for proposed fuzzy logic is defined as

$$
\begin{aligned}
R\left(A_{1}(x), A_{2}(y)\right) & =P \times V \rightarrow U \times Q=\int_{U \times V} \mu_{P}(u) /(u, v) \rightarrow \int_{U \times V} \mu_{Q}(v) /(u, v) \\
& =\int_{U \times V}\left(\mu_{P}(u) \rightarrow \mu_{Q}(v)\right) /(u, v) .
\end{aligned}
$$

Given (3.1) expression (7.3) looks like

$$
\mu_{P}(u) \rightarrow \mu_{Q}(v)=\left\{\begin{array}{l}
\left(1-\mu_{P}(u)\right) \cdot \mu_{Q}(v), \mu_{P}(u)>\mu_{Q}(v), \\
1, \mu_{P}(u) \leq \mu_{Q}(v) .
\end{array}\right.
$$

It is well known that given a unary relationship $R\left(A_{1}(x)\right)=P$ one can obtain the consequence $R\left(A_{2}(y)\right)$ by applying compositional rule of inference (CRI) to $R\left(A_{1}(x)\right)$ and $R\left(A_{1}(x), A_{2}(y)\right)$ of Type (7.3):

$$
\begin{aligned}
R\left(A_{2}(y)\right) & =P \circ R\left(A_{1}(x), A_{2}(y)\right) \\
& =\int_{U} \mu_{P}(u) / u \circ \int_{U \times V} \mu_{p}(u) \rightarrow \mu_{Q}(v) /(u, v) \\
& =\int_{V} \bigcup_{u \in U}\left[\mu_{P}(u) \wedge\left(\mu_{P}(u) \rightarrow \mu_{Q}(v)\right)\right] / v .
\end{aligned}
$$

In order that Criterion I is satisfied, that is $R\left(A_{2}(y)\right)=Q$ from (7.5) the equality

$$
\bigcup_{u \in U}\left[\mu_{P}(u) \wedge\left(\mu_{P}(u) \rightarrow \mu_{Q}(v)\right)\right]=\mu_{Q}(v)
$$


must be satisfied for arbitrary $v$ in $V$ and in order that the equality (7.6) is satisfied, it is necessary that the inequality

$$
\mu_{P}(u) \wedge\left(\mu_{P}(u) \rightarrow \mu_{Q}(v)\right) \leq \mu_{Q}(v)
$$

holds for arbitrary $u \in U$ and $v \in V$. Let us define new methods of fuzzy conditional inference of the following type:

Ant 1: If $x$ is $P$ then $y$ is $Q$

Ant2: $x$ is $P^{\prime}$

Cons: $y$ is $Q^{\prime}$.

Where $P, P \subseteq U$ and $Q, Q \subseteq V$, which requires the satisfaction of Criteria I-IV from Appendix. It is clear that (6.8) is translated by Expression (7.5), and $R\left(A_{2}(y)\right)=Q^{\prime}$ into (7.8).

\section{Theorem 2.}

If fuzzy sets $P \subseteq U$ and $Q \subseteq V$ are defined as (7.1) and (7.2) respectively and $R\left(A_{1}(x), A_{2}(y)\right)$ is defined by the following

$$
\begin{aligned}
R\left(A_{1}(x), A_{2}(y)\right) & =P \times V \underset{L 5}{\rightarrow} U \times Q=\int_{U \times V} \mu_{P}(u) /(u, v) \underset{L 5}{\rightarrow} \int_{U \times V} \mu_{Q}(v) /(u, v) \\
& =\int_{U \times V}\left(\mu_{P}(u) \underset{L 5}{\rightarrow} \mu_{Q}(v)\right) /(u, v),
\end{aligned}
$$

where

$$
\mu_{P}(u) \underset{L 5}{\rightarrow} \mu_{Q}(v)=\left\{\begin{array}{l}
\left(1-\mu_{P}(u)\right) \cdot \mu_{Q}(v), \mu_{P}(u)>\mu_{Q}(v), \\
1, \mu_{P}(u) \leq \mu_{Q}(v) .
\end{array}\right.
$$

then Criteria I, II, III and IV-1 [32] are satisfied.

Proof:

For Criteria I-III let $R\left(A_{1}(x)\right)=P^{\alpha}(\alpha>0)$ then

$$
\begin{gathered}
R\left(A_{2}(y)\right)=P^{\alpha} \circ R\left(A_{1}(x), A_{2}(y)\right) \\
=\int_{U} \mu_{P}^{\alpha}(u) / u \circ \int_{U \times V} \mu_{p}(u) \underset{L 5}{\rightarrow} \mu_{Q}(v) /(u, v) \\
=\int_{V} \bigcup_{u \in U}\left[\mu_{P}^{\alpha}(u) \wedge\left(\mu_{P}(u) \underset{L 5}{\rightarrow} \mu_{Q}(v)\right)\right] / v . \\
\exists U_{1}, U_{2} \subset U\left|U_{1} \cup U_{2}=U ;\right| U_{1} \cap U_{2}=\varnothing \\
\Rightarrow \forall u \in U_{1}\left|\mu_{P}(u)>\mu_{Q}(v) ; \forall u \in U_{2}\right| \mu_{P}(u) \leq \mu_{Q}(v) .
\end{gathered}
$$

From (7.10) and given subsets from (7.11) we have

$$
\begin{aligned}
& R\left(A_{2}(y)\right) \\
& =\left[\int_{V} \bigcup_{u \in U_{1}}\left[\mu_{P}^{\alpha}(u) \wedge\left(1-\mu_{P}(u)\right) \cdot \mu_{Q}(v)\right] / v\right] \vee\left[\int_{V} \bigcup_{u \in U_{2}}\left[\mu_{P}^{\alpha}(u) \wedge 1\right] / v\right]
\end{aligned}
$$

Let us introduce the following function (as a part of implication operation)

$$
f(u, v)=\left(1-\mu_{P}(u)\right) \cdot \mu_{Q}(v)
$$

Then the following is taking place: 


$$
\begin{aligned}
& \forall u \in U_{1} \mid \mu_{P}^{\alpha}(u) \wedge f(u, v)=\left\{\begin{array}{l}
\mu_{P}^{\alpha}(u), \mu_{P}^{\alpha}(u) \leq f(u, v), \\
f(u, v), \mu_{P}^{\alpha}(u)>f(u, v),
\end{array}\right. \\
& \forall u \in U_{2} \mid \mu_{P}^{\alpha}(u) \wedge 1=\mu_{P}^{\alpha}(u),
\end{aligned}
$$

From (7.14) and (7.15) we have

$$
(7.12)=\left[\int_{V} \bigcup_{u \in U_{2}} \mu_{P}^{\alpha}(u) / v\right]=\int_{V} \mu_{Q}^{\alpha}(v) / v=Q^{\alpha}
$$

For Criteria IV-2 [19] let $R\left(A_{1}(x)\right)=\neg P=1-P$ then

$$
\begin{aligned}
R\left(A_{2}(y)\right) & =\neg P \circ R\left(A_{1}(x), A_{2}(y)\right) \\
& =\int_{U}\left(1-\mu_{P}(u)\right) / u \circ \int_{U \times V} \mu_{p}(u) \underset{L 5}{\rightarrow} \mu_{Q}(v) /(u, v) \\
& =\int_{V} \bigcup_{u \in U}\left[\left(1-\mu_{P}(u)\right) \wedge\left(\mu_{P}(u) \underset{L 5}{\rightarrow} \mu_{Q}(v)\right)\right] / v .
\end{aligned}
$$

From (7.16) and given subsets from (7.11) we have

$$
\begin{aligned}
& R\left(A_{2}(y)\right) \\
= & {\left[\int_{V} \bigcup_{u \in U_{1}}\left[\left(1-\mu_{P}(u)\right) \wedge\left(1-\mu_{P}(u)\right) \cdot \mu_{Q}(v)\right] / v\right] \vee\left[\int_{V} \bigcup_{u \in U_{2}}\left[\left(1-\mu_{P}(u)\right) \wedge 1\right] / v\right](7.17} \\
= & {\left[\int_{V} \bigcup_{u \in U_{1}}\left[\left(1-\mu_{P}(u)\right) \wedge f(u, v)\right] / v\right] \vee\left[\int_{V} \bigcup_{u \in U_{2}}\left[\left(1-\mu_{P}(u)\right) \wedge 1\right] / v\right] . }
\end{aligned}
$$

Apparently the following is taking place

$$
\left[\left(1-\mu_{P}(u)\right) \wedge f(u, v)\right] \leq\left[\left(1-\mu_{P}(u)\right) \wedge 1\right]
$$

therefore

$$
(5.17)=\left[\int_{V} \bigcup_{u \in U_{2}}\left[\left(1-\mu_{P}(u)\right) \wedge 1\right] / v\right]=\left[\int_{V}\left(1-\mu_{Q}(v)\right) / v\right]=\neg Q \quad \text { (Q.E.D.) }
$$

Theorem 3.

If fuzzy sets $P \subseteq U$ and $Q \subseteq V$ are defined as (7.1) and (7.2) respectively and $R\left(A_{1}(x), A_{2}(y)\right)$ is defined by the following

$$
\begin{aligned}
& R\left(A_{1}(x), A_{2}(y)\right)=(P \times V \underset{L 5}{\rightarrow} U \times Q) \cap(\neg P \times V \underset{L 5}{\rightarrow} U \times \neg Q) \\
& =\int_{U \times V}\left(\mu_{P}(u) \underset{L 5}{\rightarrow} \mu_{Q}(v)\right) \wedge\left(\left(1-\mu_{P}(u)\right) \underset{L 5}{\rightarrow}\left(1-\mu_{Q}(v)\right) /(u, v) .\right.
\end{aligned}
$$

where

$$
\begin{gathered}
\left(\mu_{P}(u) \underset{L 5}{\rightarrow} \mu_{Q}(v)\right) \wedge\left(\left(1-\mu_{P}(u)\right) \underset{L 5}{\rightarrow}\left(1-\mu_{Q}(v)\right)\right) \\
=\left\{\begin{array}{l}
\left(1-\mu_{P}(u)\right) \cdot \mu_{Q}(v), \mu_{P}(u)>\mu_{Q}(v), \\
1, \mu_{P}(u)=\mu_{Q}(v), \\
\left(1-\mu_{Q}(u)\right) \cdot \mu_{P}(v), \mu_{P}(u)<\mu_{Q}(v) .
\end{array}\right.
\end{gathered}
$$

Then Criteria I, II, III and IV-2 [32] are satisfied.

Proof:

$$
\exists U_{1}, U_{2}, U_{3} \subset U\left|U_{1} \cup U_{2} \cup U_{3}=U ;\right| U_{1} \cap U_{2} \cap U_{3}=\varnothing
$$




$$
\begin{aligned}
\Rightarrow & \forall u \in U_{1} \mid \mu_{P}(u)>\mu_{Q}(v) ; \\
& \forall u \in U_{2}\left|\mu_{P}(u)=\mu_{Q}(v), \forall u \in U_{3}\right| \mu_{P}(u)<\mu_{Q}(v) .
\end{aligned}
$$

Let us introduce the following functions

$$
h_{1}(u, v)=\left(1-\mu_{P}(u)\right) \cdot \mu_{Q}(v), h_{2}(u, v)=\left(1-\mu_{Q}(u)\right) \cdot \mu_{P}(v) .
$$

Therefore from (7.18)-(7.20) for Criteria I-III let $R\left(A_{1}(x)\right)=P^{\alpha}(\alpha>0)$ then

$$
\begin{aligned}
& R\left(A_{2}(y)\right)=P^{\alpha} \circ R\left(A_{1}(x), A_{2}(y)\right) \\
& =\int_{U} \mu_{P}^{\alpha}(u) / u \circ \int_{U \times V}\left(\mu_{P}(u) \underset{L 5}{\rightarrow} \mu_{Q}(v)\right) \wedge\left(\left(1-\mu_{P}(u)\right) \underset{L 5}{\rightarrow}\left(1-\mu_{Q}(u)\right)\right) /(u, v) \\
& =\int_{V} \bigcup_{u \in U}\left[\mu_{P}^{\alpha}(u) \wedge\left(\mu_{P}(u) \underset{L 5}{\rightarrow} \mu_{Q}(v)\right) \wedge\left(\left(1-\mu_{P}(u)\right) \underset{L 5}{\rightarrow}\left(1-\mu_{Q}(u)\right)\right)\right] / v .
\end{aligned}
$$

From (7.20), (7.21) and given subsets from (7.19) we have

$$
\begin{aligned}
R\left(A_{2}(y)\right)= & {\left[\int_{V} \bigcup_{u \in U_{1}}\left[\mu_{P}^{\alpha}(u) \wedge h_{1}(u, v)\right] / v\right] \vee\left[\int_{V u \in U_{2}}\left[\mu_{P}^{\alpha}(u) \wedge 1\right] / v\right] } \\
& \vee\left[\int_{V u \in U_{3}}\left[\mu_{P}^{\alpha}(u) \wedge h_{2}(u, v)\right] / v\right] .
\end{aligned}
$$

Then again the following is taking place:

$$
\begin{gathered}
\forall u \in U_{1} \mid \mu_{P}^{\alpha}(u) \wedge h_{1}(u, v)=\left\{\begin{array}{l}
\mu_{P}^{\alpha}(u), \mu_{P}^{\alpha}(u) \leq h_{1}(u, v), \\
h_{1}(u, v), \mu_{P}^{\alpha}(u)>h_{1}(u, v),
\end{array}\right. \\
\forall u \in U_{2} \mid \mu_{P}^{\alpha}(u) \wedge 1=\mu_{P}^{\alpha}(u), \\
\forall u \in U_{3} \mid \mu_{P}^{\alpha}(u) \wedge h_{2}(u, v)=\left\{\begin{array}{l}
\mu_{P}^{\alpha}(u), \mu_{P}^{\alpha}(u) \leq h_{2}(u, v), \\
h_{2}(u, v), \mu_{P}^{\alpha}(u)>h_{2}(u, v),
\end{array}\right.
\end{gathered}
$$

From (7.23) - (7.25) we have

$$
\text { (7.21) }=\left[\int_{V} \bigcup_{u \in U_{2}} \mu_{P}^{\alpha}(u) / v\right]=\int_{V} \mu_{Q}^{\alpha}(v) / v=Q^{\alpha}
$$

For Criteria IV-2 let $R\left(A_{1}(x)\right)=\neg P=1-P$ then

$$
\begin{aligned}
& R\left(A_{2}(y)\right)=\neg P \circ R\left(A_{1}(x), A_{2}(y)\right) \\
& =\int_{U}\left(1-\mu_{P}(u)\right) / u \circ \int_{U \times V}\left(\mu_{P}(u) \underset{L 5}{\rightarrow} \mu_{Q}(v)\right) \wedge\left(\left(1-\mu_{P}(u)\right) \underset{L 5}{\rightarrow}\left(1-\mu_{Q}(v)\right)\right) /(u, v)(7.26) \\
& =\int_{V} \bigcup_{u \in U}\left[\left(\mu_{P}(u) \underset{L 5}{\rightarrow} \mu_{Q}(v)\right) \wedge\left(\left(1-\mu_{P}(u)\right) \underset{L 5}{\rightarrow}\left(1-\mu_{Q}(v)\right)\right)\right] / v .
\end{aligned}
$$

From (7.22), (7.26) and given subsets from (7.19) we have

$$
\begin{aligned}
R\left(A_{2}(y)\right)= & {\left[\int_{V u \in U_{1}}\left[\left(1-\mu_{P}(u)\right) \wedge h_{1}(u, v)\right] / v\right] \vee\left[\int_{V u \in U_{2}}\left[\left(1-\mu_{P}(u)\right) \wedge 1\right] / v\right] } \\
& \vee\left[\int_{V u \in U_{3}}\left[\left(1-\mu_{P}(u)\right) \wedge h_{2}(u, v)\right] / v\right] .
\end{aligned}
$$

Apparently the following is taking place 


$$
\left[\left(1-\mu_{P}(u)\right) \wedge h_{1}(u, v)\right] \leq\left[\left(1-\mu_{P}(u)\right) \wedge 1\right]
$$

and

$$
\left[\left(1-\mu_{P}(u)\right) \wedge h_{2}(u, v)\right] \leq\left[\left(1-\mu_{P}(u)\right) \wedge 1\right]
$$

therefore

$$
(7.27)=\left[\int_{V} \bigcup_{u \in U_{2}}\left[\left(1-\mu_{P}(u)\right) \wedge 1\right] / v\right]=\left[\int_{V}\left(1-\mu_{Q}(u)\right) / v\right]=\neg Q \quad \text { (Q.E.D.) }
$$

For many real practical applications a decision making apparatus could be based not on a fuzzy conditional proposition of the type: "If $x$ is $P$ then $y$ is $Q$ ", but rather on a rule of the following type

$$
\text { "If } x_{1} \text { is } P_{1} \text { and } x_{2} \text { is } P_{2} \text { and } \cdots \text { and } x_{n} \text { is } P_{n} \text { then } y \text { is } Q \text { " }
$$

And correspondent fuzzy sets are represented as such that

$$
\forall i=\overline{1, n} \Rightarrow P_{i} \subset U\left|\mu_{P_{i}}: U \rightarrow[0,1], Q \subset V\right| \mu_{Q}: V \rightarrow[0,1]
$$

Given (7.1) and (7.2) a binary relationship for a fuzzy conditional proposition of the Type (7.28) for proposed fuzzy logic is defined as

$$
D=R\left(A_{1}(x), A_{2}(y)\right)=\left[\bigcap_{i} P_{i}\right] \times V \underset{L 5}{\rightarrow} U \times Q
$$

Theorem 4. If a fuzzy conditional proposition is defined as (7.28), correspondent fuzzy sets of antecedents and consequent are presented as (7.29) and a binary relationship for a fuzzy conditional proposition is from (7.30), and "elementary" binary relationships are defined as following

$$
\forall i=\overline{1, n} \mid D_{i}=R_{i}\left(A_{1}(x), A_{2}(y)\right)=P_{i} \times V \underset{L 5}{\rightarrow} U \times Q
$$

then the following expression is taking place

$$
D=D_{1} \cup D_{2} \cup \cdots \cup D_{n}=\bigcup_{i} D_{i}
$$

and as a result the following is also true.

$$
\left[\bigcap_{i} P_{i}\right] \times V \underset{L 5}{\rightarrow} U \times Q=\bigcup_{i}\left(P_{i} \times V \underset{L 5}{\rightarrow} U \times Q\right)
$$

Proof:

Let $\exists U_{1}, U_{2} \subset U\left|U_{1} \cup U_{2}=U ;\right| U_{1} \cap U_{2}=\varnothing, \quad \forall u \in U_{1} \mid{ }_{i} \mu_{P_{i}}(u) \leq \mu_{Q}(v)$; $\forall u \in U_{2} \mid{ }_{i} \mu_{P_{i}}(u)>\mu_{Q}(v)$ and let us denote each

$$
D_{i}=\left\{\begin{array}{l}
D_{i}^{I}, \forall u \in U_{1}^{\prime} \\
D_{i}^{I I}, \forall u \in U_{2}^{\prime}
\end{array} \Rightarrow D_{i}^{I}=1 \mid \mu_{P_{i}}(u) \leq \mu_{Q}(v)\right.
$$

whereas

$$
D^{I}=\left.1\right|_{i} \mu_{P_{i}}(u) \leq \mu_{Q}(v)
$$

From (7.34)

$$
D^{I}=\bigcup_{i} D_{i}^{I}
$$


Since

$$
\begin{aligned}
D_{i}^{I I} & =\left.\left(1-\hat{i}_{i} \mu_{P}(u)\right) \cdot \mu_{Q}(v)\right|_{i} \mu_{P_{i}}(u)>\mu_{Q}(v) \\
& =\left.\vee_{i} \mu_{P_{i}}(u) \cdot \mu_{Q}(v)\right|_{i} \mu_{P_{i}}(u)>\mu_{Q}(v)
\end{aligned}
$$

From (7.35)

$$
D^{I I}=\bigcup_{i} D_{i}^{I I}
$$

Both (7.35) and (7.37) prove (7.33)

Let us consider more complex fuzzy conditional proposition of the type:

$$
\begin{aligned}
R_{i}= & \text { "If } x_{1} \text { is } P_{i k 1} \text { and } x_{2} \text { is } P_{i k 2} \text { and } \cdots \text { and } x_{n} \text { is } P_{i k n} \\
& \text { then } y \text { is } Q_{i l} \text {, else } y \text { is not } Q_{i l} \text { " }
\end{aligned}
$$

For

$$
i=\overline{1, N}, k=\overline{1, \operatorname{CardU}}, l=\overline{1, \operatorname{CardV}}
$$

where $P_{i k j} \subseteq U, j=\overline{1, n}, Q_{i l} \subseteq V$ are normal fuzzy sets of a type (7.1) and (7.2)

$$
P_{i k j}=\int_{U} \mu_{P_{i k j}}(u) / u, Q_{i l}=\int_{V} \mu_{Q_{i l}}(v) / v
$$

with unimodal membership functions $\mu_{P_{i k j}}(u): U \rightarrow[0,1], \mu_{Q_{i l}}(u): V \rightarrow[0,1]$ and $U, V \subset \mathfrak{R}^{+}$are countable, finite universes of discourses, i.e.

$\operatorname{Card} U=\operatorname{CardV}=M$. Note that a unimodality of (7.39) means that for all singletons $\mu_{P_{i k j}}(u) / u, \mu_{Q_{i l}}(v) / v$ the following is taking place

$$
\exists ! u^{\prime} \in U\left|\mu_{P_{i k j}}\left(u^{\prime}\right)=1 ; \forall u \neq u^{\prime} \in U\right| \mu_{P_{i k j}}(u)<1,
$$

and

$$
\exists ! v^{\prime} \in V\left|\mu_{Q_{i l}}\left(v^{\prime}\right)=1 ; \forall v \neq v^{\prime} \in V\right| \mu_{Q_{i l}}(v)<1
$$

Note that each rule of type (7.38) looks like that

$$
\begin{aligned}
& R_{i}\left(A_{1}(x), A_{2}(y)\right) \\
& =\left[\left(\bigcap_{j} P_{i k j}\right) \times V \underset{L 5}{\rightarrow} U \times Q_{i l}\right] \cap\left[\neg\left(\bigcap_{j} P_{i k j}\right) \times V \underset{L 5}{\rightarrow} U \times \neg Q_{i l}\right], \\
& j=\overline{1, n} ; k, l=\overline{1, M} ; i=\overline{1, N}
\end{aligned}
$$

Let us point to the fact that (7.40) is a mathematical representation of the following fuzzy conditional rule:

Ant 1:

If $x_{1}$ is $P_{i k 1}$ and $x_{2}$ is $P_{i k 2}$ and $\cdots$ and $x_{n}$ is $P_{i k n}$ then $y$ is $Q_{i l}$, else $y$ is not $Q_{i l}$

Ant2: $x_{1}$ is $P_{i k 1}^{\prime}$ and $x_{2}$ is $P_{i k 2}^{\prime}$ and $\cdots$ and $x_{n}$ is $P_{i k n}^{\prime}$

Cons: $y$ is $Q_{i l}^{\prime}$,

In traditional way (7.41) looks like:

$$
Q_{i l}^{\prime}=\left[\bigcap_{j} P_{i k j}^{\prime}\right] \circ R_{i}\left(A_{1}(x), A_{2}(y)\right), j=\overline{1, n} ; k, l=\overline{1, M} ; i=\overline{1, N},
$$


But for $j=\overline{1, n}$ we have

$$
\begin{aligned}
& \int_{V} \mu_{Q_{i l}^{\prime}}(v) / v \\
& =\int_{U} \bigcap_{j} \mu_{P_{i k j}^{\prime}}(u) / u \circ \int_{U \times V}\left(\bigcap_{j} \mu_{P_{i k j}}(u) \underset{L 5}{\rightarrow} \mu_{Q_{i l}}(v)\right) \wedge\left(\left(1-\bigcap_{j} \mu_{P_{i k j}}(u)\right) \underset{L 5}{\rightarrow}\left(1-\mu_{Q_{i l}}(v)\right)\right) /(u, v) \\
& =\bigcup_{j} \int_{V} \bigcup_{u \in U}\left[\mu_{P_{i k j}^{\prime}}(u) \wedge\left(\mu_{P_{i k j}}(u) \underset{L 5}{\rightarrow} \mu_{Q_{i l}}(v)\right) \wedge\left(\left(1-\mu_{P_{k j j}}(u)\right) \underset{L 5}{\rightarrow}\left(1-\mu_{Q_{i l}}(v)\right)\right)\right] / v .
\end{aligned}
$$

Let us define

$$
\mu_{R_{i}}(u, v)=\left[\left(\mu_{P_{i k}}(u) \underset{L 5}{\rightarrow} \mu_{Q_{i l}}(v)\right) \wedge\left(\left(1-\mu_{P_{k j}}(u)\right) \underset{L 5}{\rightarrow}\left(1-\mu_{Q_{i l}}(v)\right)\right)\right] / v
$$

from (7.43) we get

$$
\int_{V} \mu_{Q_{i l}^{\prime}}(v) / v=\int_{V} \bigcup_{u \in U} \mu_{P_{i k j}^{\prime}}(u) / u \wedge \mu_{R_{i}}(u, v) / v
$$

Suppose

$$
\left.U=U_{1} \cup U_{2} \mid U_{1} \cap U_{2}=\varnothing, \text { and } \begin{array}{l}
\forall u \in U_{1} \mid \mu_{P_{k j}}(u) \neq \mu_{Q_{l}}(v) \\
\forall u \in U_{2} \mid \mu_{P_{k j}}(u)=\mu_{Q_{l}}(v)
\end{array}\right\}
$$

Proposition 4. For discussed fuzzy logic a fuzzy conditional rule of Type (7.41) satisfies the following feature

$$
\forall u \in U_{2} \mid\left[\mu_{P_{i k j}}(u)=\mu_{Q_{i l}}(v)\right] \Rightarrow \mu_{R_{i}}^{*}(u, v)=\sup _{U \times V} \mu_{R_{i}}(u, v)=1 ;
$$

Let us call the binary relationship matrix $R_{i}\left(A_{1}(x), A_{2}(y)\right), i=\overline{1, N}$, from (7.42) by "elementary knowledge" $(E K)$. Each EK is characterized by the following features

$$
U^{I I} \subseteq U=\left\{\forall r, p \in K=\{k\} ; u_{r}, u_{p} \in U \mid \mu_{P_{i k j}}\left(u_{k}\right)=\mu_{P_{i k j}}\left(u_{p}\right)\right\}^{*}
$$

Same is also true for $V^{I I} \subseteq V$. Since both membership functions $\mu_{P_{i k j}}(u): U \rightarrow[0,1], \mu_{Q_{i l}}(u): V \rightarrow[0,1]$ are unimodal, then $\left.\operatorname{Card}^{I I}\right|^{\max }=\frac{M-1}{2}$ and $\left.\operatorname{Card} U^{I I}\right|^{\min }=0$. (The same is true for $\operatorname{CardV}^{I I}$ ).

It is apparent that both $U^{I I}=\varnothing, V^{I I}=\varnothing$. In practical terms it means that if $U^{I I}=\varnothing$, then either

$$
\mu_{P_{i k j}}\left(u_{1}\right)=\sup _{\forall u_{k} \in U} \mu_{P_{i k j}}\left(u_{k}\right) ; \mu_{P_{i k j}}\left(u_{M}\right)=\inf _{\forall u_{k} \in U} \mu_{P_{i k j}}\left(u_{k}\right)
$$

or

$$
\mu_{P_{i k j}}\left(u_{1}\right)=\inf _{\forall u_{k} \in U} \mu_{P_{i k j}}\left(u_{k}\right) ; \mu_{P_{i k j}}\left(u_{M}\right)=\sup _{\forall u_{k} \in U} \mu_{P_{i k j}}\left(u_{k}\right)
$$

whereas if $V^{I I}=\varnothing$, then either

$$
\mu_{Q_{i l}}\left(v_{1}\right)=\sup _{\forall v_{l} \in V} \mu_{Q_{i l}}\left(v_{l}\right) ; \mu_{Q_{i l}}\left(v_{M}\right)=\inf _{\forall v_{l} \in V} \mu_{Q_{i l}}\left(v_{l}\right)
$$

or

$$
\mu_{Q_{i l}}\left(v_{1}\right)=\inf _{\forall v_{l} \in V} \mu_{Q_{i l}}\left(v_{l}\right) ; \mu_{Q_{i l}}\left(v_{M}\right)=\sup _{\forall v_{l} \in V} \mu_{Q_{i l}}\left(v_{l}\right)
$$


Definition 7. The $E K$ of the type $R_{i}\left(A_{1}(x), A_{2}(y)\right), i=\overline{1, N}$, from (7.42) is called logically contradictive or fruitless if a membership function of a consequent in fuzzy conditional rule (7.41) is non-unimodal or when two different antecedents induct the same consequent.

Given (7.46)-(7.48), based on defined earlier $U^{I I}, V^{I I}$ sets and taking into account that $R_{i}\left(A_{1}(x), A_{2}(y)\right)=\left\|\mu_{R_{i}}\left(u_{k}, v_{l}\right)\right\|, \quad k, l=\overline{1, M} ; i=\overline{1, N}$ suppose that

$$
U^{I I} \neq \varnothing, V^{I I} \neq \varnothing
$$

then

$$
\begin{gathered}
\exists u^{*} \in U ; \exists v^{*}, v^{* *} \in V ; v^{*} \neq v^{* *} \mid\left[\mu_{P_{i k j}}\left(u^{*}\right)=\mu_{Q_{i l}}\left(v^{*}\right)=\mu_{Q_{i l}}\left(v^{* *}\right)\right] \\
\Rightarrow \mu_{R_{i}}\left(u^{*}, v^{*}\right)=\mu_{R_{i}}\left(u^{*}, v^{* *}\right)=\sup _{\forall u_{k} \in U} \mu_{R_{i}}(u, v)=1 \\
\exists v^{I} \in V ; \exists u^{I}, u^{I I} \in U ; u^{I} \neq u^{I I} \mid\left[\mu_{Q_{i l}}\left(v^{I}\right)=\mu_{P_{i k j}}\left(u^{I}\right)=\mu_{P_{i k j}}\left(u^{I I}\right)\right] \\
\Rightarrow \mu_{R_{i}}\left(u^{I}, v^{I}\right)=\mu_{R_{i}}\left(u^{I I}, v^{I}\right)=\sup _{\forall v_{l} \in V} \mu_{R_{i}}(u, v)=1
\end{gathered}
$$

${ }^{*}$ From now on upper and lower indices of based variables $\mathrm{u}$ and $\mathrm{v}$ denote not a membership functions, but their correspondent singletons

It is clear, that for $U^{2}$ from (7.45)

$$
\begin{aligned}
& \left.\operatorname{CardU}^{I I}\right|^{\max }=\left.\operatorname{CardV}^{I I}\right|^{\max }=\frac{M-1}{2} \\
& \left.\Rightarrow \operatorname{CardU}_{2}\right|^{\max }=2 M-1 ; \operatorname{Tr}\left[R_{i}\right]=\operatorname{Tr}\left[R_{i}^{I}\right]=M
\end{aligned}
$$

where $\operatorname{Tr}\left[R_{i}\right], \operatorname{Tr}\left[R_{i}^{I}\right]$-are traces of matrixes $R_{i}, R_{i}^{I}$ and $r_{i j}^{I}=r_{i, N+1-j} ; j=\overline{1, N}$. 1. Let $U^{I I}=\varnothing, V^{I I} \neq \varnothing$, then

$$
\begin{gathered}
\exists u^{*} \in U ; \exists v^{*}, v^{* *} \in V ; v^{*} \neq v^{* *} \mid\left[\mu_{P_{i k j}}\left(u^{*}\right)=\mu_{Q_{i l}}\left(v^{*}\right)=\mu_{Q_{i l}}\left(v^{* *}\right)\right] \\
\Rightarrow \mu_{R_{i}}\left(u^{*}, v^{*}\right)=\mu_{R_{i}}\left(u^{*}, v^{* *}\right)=\sup _{\forall u_{k} \in U} \mu_{R_{i}}(u, v)=1 \\
\left.\operatorname{Card}^{I I}\right|^{\max }=\left.\frac{M-1}{2} \Rightarrow \operatorname{Card}_{2}\right|^{\max }=M ; \operatorname{Tr}\left[R_{i}\right]=\operatorname{Tr}\left[R_{i}^{I}\right]
\end{gathered}
$$

2. Let $U^{I I} \neq \varnothing, V^{I I}=\varnothing$, then

$$
\begin{gathered}
\exists v^{I} \in V ; \exists u^{I}, u^{I I} \in U ; u^{I} \neq u^{I I} \mid\left[\mu_{Q_{i l}}\left(v^{I}\right)=\mu_{P_{i k j}}\left(u^{I}\right)=\mu_{P_{i k j}}\left(u^{I I}\right)\right] \\
\Rightarrow \mu_{R_{i}}\left(u^{I}, v^{I}\right)=\mu_{R_{i}}\left(u^{I I}, v^{I}\right)=\sup _{\forall v_{l} \in V} \mu_{R_{i}}(u, v)=1 \\
\left.\operatorname{CardU}^{I I}\right|^{\max }=\left.\frac{M-1}{2} \Rightarrow \operatorname{Card}_{2}\right|^{\max }=M ; \operatorname{Tr}\left[R_{i}\right]=\operatorname{Tr}\left[R_{i}^{I}\right]
\end{gathered}
$$

3. Let $U^{I I}=\varnothing, V^{I I}=\varnothing$, then either $\forall l=\overline{1, M} ; \exists ! r=l \in K \mid \mu_{P_{i k j}}\left(u_{r}\right)=\mu_{Q_{i l}}\left(v_{l}\right)$

$$
\Rightarrow \mu_{R_{i}}\left(u_{r}, v_{l}\right)=\sup _{(u, v) \in U \times V} \mu_{R_{i}}(u, v)=1
$$

and $\operatorname{Tr}\left[R_{i}(\cdot)\right]=M$, which means that there is a major diagonal of a matrix $R_{i}(\cdot)$ consists of singles (1), or 


$$
\begin{gathered}
\forall p=\overline{1, M} ; \exists ! l=M+1-p \in K \mid \mu_{P_{i k j}}\left(u_{p}\right)=\mu_{Q_{i l}}\left(v_{M+1-p}\right) \\
\Rightarrow \mu_{R_{i}}\left(u_{p}, v_{l}\right)=\sup _{(u, v) \in U \times V} \mu_{R_{i}}(u, v)=1
\end{gathered}
$$

which means that there is a peripheral diagonal of a matrix $R_{i}(\cdot)$ consists of singles. Taking into account (7.49)-(7.54) we put together the following

\section{Definition 8.}

If $P_{i k j} \subseteq U, j=\overline{1, n}, Q_{i l} \subseteq V, \quad i=\overline{1, N}, k=1, \overline{\operatorname{Card} U}, l=1, \overline{\operatorname{CardV} V}$ are normal fuzzy sets of a type (7.39) and the sets are defined as the follows $U^{I I} \subseteq U, V^{I I} \subseteq V \mid U^{I I}=\varnothing, V^{I I}=\varnothing$, then binary relationship matrix $R_{i}\left(A_{1}(x), A_{2}(y)\right), i=\overline{1, N}$ of type (7.40) or its counterpart $R_{i}^{I}($.$) , have major$ diagonal, which consists of singles.

Based on this definition and (7.49)-(7.53) let us formulate the following:

\section{Theorem 5.}

An $E K$ of the type $R_{i}\left(A_{1}(x), A_{2}(y)\right), i=\overline{1, N}$, from (7.42) is logically contradictive or fruitless if the following inequality is taking place

$$
\left|\operatorname{Tr}\left[R_{i}(\cdot)\right]-\operatorname{Tr}\left[R_{i}^{I}(\cdot)\right]\right| \leq \varepsilon, i=\overline{1, n}
$$

where $\varepsilon$ should be defined based on certain practical considerations, i.e. $\varepsilon=f(\operatorname{CardU})$.

\section{Proof:}

Let is

$$
\left|\operatorname{Tr}\left[R_{i}(\cdot)\right]-\operatorname{Tr}\left[R_{i}^{I}(\cdot)\right]\right|=0, i=\overline{1, n}
$$

then based on (7.42) we have

$$
\begin{aligned}
& \int_{V} \mu_{Q_{i l}^{\prime}}(v) / v \\
& =\int_{V u \in U}\left[\mu_{P_{i k j}^{\prime}}(u) \wedge\left(\mu_{P_{i k j}}(u) \underset{L 5}{\rightarrow} \mu_{Q_{i l}}(v)\right) \wedge\left(\left(1-\mu_{P_{k j j}}(u)\right) \underset{L 5}{\rightarrow}\left(1-\mu_{Q_{i l}}(v)\right)\right)\right] / v \\
& =\int_{V} \bigcup_{u \in U}\left[\mu_{P_{i k j}^{\prime}}(u) \wedge \mu_{R_{i}}(u, v)\right] / v .
\end{aligned}
$$

Let us introduce the following sets $U_{1}, U_{2} \subseteq U \mid U_{1} \cap U_{2}=\varnothing, U_{1} \cup U_{2}=U$, which are

$$
\left.\begin{array}{l}
\forall u \in U_{1} \mid \mu_{P_{i k j}}(u) \neq \mu_{Q_{i l}}(v) \Rightarrow \mu_{R_{i}}(u, v)<1, \\
\forall u \in U_{2} \mid \mu_{P_{i k j}}(u)=\mu_{Q_{i l}}(v) \Rightarrow \mu_{R_{i}}(u, v)=1
\end{array}\right\}
$$

From (7.55) and (7.56) we are getting the following

$$
(7.56)=\int_{V}\left\{\bigcup_{u \in U_{1}} \mu_{P^{\prime} k j}(u) \wedge \mu_{R_{i}}(u, v)\right\} \vee\left\{\bigcup_{u \in U_{2}} \mu_{P_{i k j}^{\prime}}(u) \wedge \mu_{R_{i}}(u, v)\right\} / v .
$$

Taking into account (7.56) we get the following:

$$
\bigcup_{u \in U_{2}} \mu_{P_{i k j}^{\prime}}(u) \wedge \mu_{R_{i}}(u, v)>\bigcup_{u \in U_{1}} \mu_{P_{i k j}^{\prime}}(u) \wedge \mu_{R_{i}}(u, v)
$$

therefore we are getting

$$
(7.56)=\left\{\bigcup_{u \in U_{2}} \mu_{P_{i k j}^{\prime}}(u) \wedge \mu_{R_{i}}(u, v)\right\} / v .
$$


Note that the set $U_{2}$ is defined by both $U^{I I}$ and $V^{I I}$. We are considering the following case first:

1) $U^{I I} \neq \varnothing, V^{I I} \neq \varnothing$. Since $P_{i k j}^{I} \subseteq U$ is normal fuzzy set, then

$\exists u^{*} \in U \mid \mu_{P_{i k j}^{\prime}}^{\prime}\left(u^{*}\right)=1$ and given (7.49) and (7.50) we get

$$
\forall u \in U_{2} ; \exists v^{*}, v^{* *} \in V ; v^{*} \neq v^{* *} \mid \mu_{R_{i}}\left(u, v^{*}\right)=\mu_{R_{i}}\left(u, v^{* *}\right)=1
$$

therefore from (7.59) we get the following $\exists v_{1}, v_{2} \in V ; v_{1} \neq v_{2} \mid \mu_{Q_{i l}^{I}}\left(v_{1}\right)=\mu_{Q_{i l}^{I}}\left(v_{2}\right)$ (here we consider not a membership functions (7.39), but their correspondent singletons) and therefore

$$
\begin{aligned}
& (7.59)=\left\{\left[\bigcup_{u \in U_{2}} \mu_{P_{i k j}^{\prime}}(u)\right] \wedge\left[\bigcup_{u \in U_{2}} \mu_{R_{i}}(u, v)\right]\right\} \\
& =\mu_{P_{i k j}^{\prime}}\left(u^{*}\right) \wedge \mu_{R_{i}}\left(u, v^{*}\right)=\mu_{P_{i k j}^{\prime}}\left(u^{*}\right) \wedge \mu_{R_{i}}\left(u, v^{* *}\right)=1 .
\end{aligned}
$$

In other words from (7.59) we see that the membership function of a Consequent from fuzzy conditional inference rule (7.41) $Q_{i l}^{I}=\int_{V} \mu_{Q_{i l}^{I}}(v) / v$ is poly modal one.

2) $U^{I I}=\varnothing, V^{I I} \neq \varnothing$. Since $P_{i k j}^{I} \subseteq U$ is normal fuzzy set, then

$\exists u^{1} \in U_{2} \mid \mu_{P_{i k j}^{\prime}}^{\prime}\left(u^{1}\right)=1$ and given (7.51) and (7.52) we get

$$
\exists u^{*} \in U_{2} ; \exists v^{*}, v^{* *} \in V ; v^{*} \neq v^{* *} \mid \mu_{R_{i}}\left(u^{*}, v^{*}\right)=\mu_{R_{i}}\left(u^{*}, v^{* *}\right)=1
$$

therefore when $u^{1}=u^{*}$ from (7.59) we get the following

$$
\begin{aligned}
& \exists v_{1}, v_{2} \in V ; v_{1} \neq v_{2} \mid \mu_{Q_{i l}^{I}}\left(v_{1}\right)=\mu_{Q_{i l}^{I}}\left(v_{2}\right)=\left\{\left[\bigcup_{u \in U_{2}} \mu_{P_{i k j}^{\prime}}(u)\right] \wedge\left[\bigcup_{u \in U_{2}} \mu_{R_{i}}(u, v)\right]\right\} \\
& =\mu_{P_{i k j}^{\prime}}\left(u^{*}\right) \wedge \mu_{R_{i}}\left(u^{*}, v^{*}\right)=\mu_{P_{i k j}^{\prime}}\left(u^{*}\right) \wedge \mu_{R_{i}}\left(u^{*}, v^{* *}\right)=1 .
\end{aligned}
$$

Here we also see that the membership function of a Consequent from fuzzy conditional inference rule (7.41) $Q_{i l}^{I}=\int_{V} \mu_{Q_{i l}^{I}}(v) / v$ is poly modal one.

3) $U^{I I} \neq \varnothing, V^{I I}=\varnothing$. Since $P_{i k j}^{I} \subseteq U$ is normal fuzzy set, then $\exists u^{1} \mid \mu_{P_{i k j}^{\prime}}^{\prime}\left(u^{1}\right)=1$ and given (7.53) and (7.54) we get

$$
\exists v^{I} \in V ; \exists u^{I}, u^{I I} \in U ; u^{I} \neq u^{I I} \mid \mu_{Q_{i l}}\left(v^{I}\right)=\mu_{P_{i k j}}\left(u^{I}\right)=\mu_{P_{i k j}}\left(u^{I I}\right)
$$

therefore from (7.59) we get the following

$$
\exists v^{I} \in V \mid \mu_{Q_{i l}^{I}}(v)=\left\{\left[\bigcup_{u \in U_{2}} \mu_{P_{i k j}^{\prime}}(u)\right] \wedge\left[\bigcup_{u \in U_{2}} \mu_{R_{i}}(u, v)\right]\right\}=\mu_{P_{i k j}^{\prime}}\left(u^{I}\right) \wedge \mu_{R_{i}}\left(u^{I}, v^{I}\right)=1
$$

In a meantime one can see that

$$
\begin{aligned}
& \exists u^{I I} \mid \mu_{P_{i k j}^{\prime}}\left(u^{I I}\right)=1 \Rightarrow \mu_{Q_{i l}^{I}}\left(v^{I}\right)=\left\{\left[\bigcup_{u \in U_{2}} \mu_{P_{i k j}^{\prime}}(u)\right] \wedge\left[\bigcup_{u \in U_{2}} \mu_{R_{i}}(u, v)\right]\right\} \\
& =\mu_{P_{i k j}^{\prime}}\left(u^{I I}\right) \wedge \mu_{R_{i}}\left(u^{I I}, v^{I}\right)=1
\end{aligned}
$$

In other words we see that there are two fuzzy sets $P_{i k j}^{I}, P_{i k j}^{I I} \subseteq U \mid P_{i k j}^{I} \neq P_{i k j}^{I I}$, when used as an Antecedents in fuzzy conditional inference rule (7.41), induce the same Consequent, in other words, based on the Definition 7 we have logically contradictive EK from (7.42).

(Q.E.D.) 
Based on the results of this Theorem we have to present the following

\section{Corollary.}

In order to make $E K$ from (7.42) logically non-contradictive, both membership functions of a fuzzy sets $P_{i k j} \subseteq U, j=\overline{1, n}, Q_{i l} \subseteq V$ from (7.39) be unimodaland $U^{I I}, V^{I I}$, defined in (7.47) and (7.48) correspondingly, would be empty, i.e. $U^{I I}=\varnothing, V^{I I}=\varnothing$.

\section{Concluding Remarks}

In this paper we proposed new t-norm fuzzy logic in which: 1) Truth values of an implication operator are based on truth values of both antecedent and consequent, 2) Implication operator could be considered as one of the fuzziest implication from the list [34] of known so far; 3) The suggested implication operator is a base for fuzzy conditional inference rules and satisfies the set of important human intuition Criteria; 4) Important features of these rules are investigated.

\section{References}

[1] Aliev, R.A. and Tserkovny, A. (1988) The Knowledge Representation in Intelligent Robots Based on Fuzzy Sets. Doklady Mathematics, 37, 541-544.

[2] Aliev, R.A., Mamedova, G.A. and Tserkovny, A.E. (1991) Fuzzy Control Systems. Energoatomizdat, Moscow.

[3] Aliev, R.A., Fazlollahi, B. and Aliev, R.R. (2004) Soft Computing and Its Application in Business and Economics. Physica-Verlag, Springer, Berlin, Heidelberg. https://doi.org/10.1007/978-3-540-44429-9

[4] Aliev, R.A. and Tserkovny, A. (2011) Systemic Approach to Fuzzy Logic Formalization for Approximate Reasoning. Information Sciences, 181, 1045-1059.

[5] Tserkovny, A. (2016) Fuzzy Logic for Incidence Geometry. The Scientific World Journal, 2016, Article ID: 9057263. https://doi.org/10.1155/2016/9057263

[6] Bandler, W. and Kohout, L.J. (1980) Fuzzy Relational Products as a Tool for Analysis of Complex Artificial and Natural Systems. In: Wang, P.P. and Chang, S.K., Eds., Fuzzy Sets. Theory and Applications to Policy Analysis and Information Systems, Plenum Press, New York, 311-367.

[7] Bandler, W. and Kohout, L.J. (1980) The Identification of Hierarchies in Symptoms and Patients through Computation of Fuzzy Relational Products. In: Parslow, R.D., Ed., BCS 81: Information Technology for the Eighties, Heyden \& Sons, 191-194.

[8] Bandler, W. and Kohout, L.J. (1980) Semantics of Fuzzy Implication Operators and Relational Products. International Journal of Man-Machine Studies, 12, 89-116.

[9] Bandler, W. and Kohout, L.J. (1980) Fuzzy Power Sets and Fuzzy Implication Operators. Fuzzy Sets and Systems, 4, 13-30.

[10] Bandler, W. and Kohout, L.J. (1984) The Four Modes of Inference in Fuzzy Expert Systems. In: Trappl, R., Ed., Cybernetics and Systems Research 2, North Holland, Amsterdam, 97-104.

[11] Bandler, W. and Kohout, L.J. (1985) Probabilistic vs. Fuzzy Production Rules in Expert Systems. International Journal of Man-Machine Studies, 22, 347-353.

[12] Buckley, J. and Siler, W. (1999) $L_{\infty}$ Fuzzy Logic. Fuzzy Sets and Systems, 107, 309-322. 
[13] Coupland, S. and John, R. (2008) Type-2 Fuzzy Logic, Modeling Uncertainty. In: Bustince, H., Herrera, F. and Montero, J., Eds., Fuzzy Sets and Their Extensions. Representation, Aggregation and Models, Vol. 220, Springer, Berlin, Heidelberg, 3-22. https://doi.org/10.1007/978-3-540-73723-0_1

[14] Fedrizzi, M. and Fuller, R. (1992) Stability in Possibilistic Linear Programming Problems with Continuous Fuzzy Number Parameters. Fuzzy Sets and Systems, 47, 187-191.

[15] Franksen, O.I. (1978) Group Representation of Finite Polyvalent Logic. Proceedings of the 7 th Triennial International Federation of Automatic Control World Congress, Pergamon, IFAC, Helsinki.

[16] Fukami, S., Mizumoto, M. and Tanaka, K. (1980) Some Considerations of Fuzzy Conditional Inference. Fuzzy Sets and Systems, 4, 243-273.

[17] Fuller, R. and Zimmermann, H.-J. (1993) On Zadeh's Compositional Rule of Inference. In: Lowen, R. and Roubens, M., Eds., Fuzzy Logic. State of the Art, Theory and Decision Library, Series D, Kluwer Academic Publisher, Dordrecht, 193-200. https://doi.org/10.1007/978-94-011-2014-2_19

[18] Gerhke, M., Walker, C.L. and Walker, E.A. (2003) Normal Forms and Truth Tables for Fuzzy Logics. Fuzzy Sets and Systems, 138, 25-51.

[19] Jantzen, J. (1995) Array Approach to Fuzzy Logic. Fuzzy Sets and Systems, 70, 359-370.

[20] Jenei, S. (1999) Continuity in Zadeh's Compositional Rule of Inference. Fuzzy Sets and Systems, 104, 333-339.

[21] Kandel, A. and Last, M. (2007) Special Issue on Advances in Fuzzy Logic. Information Sciences, 177, 329-331.

[22] Kallala, M. and Kohout, L.J. (1984) The Use of Fuzzy Implication Operators in Clinical Evaluation of Neurological Movement Disorders. International Symposium on Fuzzy Information Processing in Artificial Intelligence and Operational Research, Christchurch College, Cambridge University.

[23] Kallala, M. and Kohout, L.J. (1986) A 2-Stage Method for Automatic Handwriting Classification by Means of Norms and Fuzzy Relational Inference. Proceedings of NAFIPS 86, NAFIPS Congress, New Orleans.

[24] Karnik, N.J. and Mendel, M. (2001) Operations on Type-2 Fuzzy Sets. Fuzzy Sets and Systems, 122, 327-348.

[25] Kiszka, J.B., Kochanska, M.E. and Sliwinska, D.S. (1985) The Influence of Some Fuzzy Implication Operators on the Accuracy of a Fuzzy Model. Fuzzy Sets and Systems, 15, Part1, 111-128; Part2, 223-240.

[26] Kohout, L.J. and Bandler, W. (1985) Relational-Product Architecture for Information Processing. Expert Systems Information Science, 37, 25-37.

[27] Kohout, L.J., Eds. (1986) Perspectives on Intelligent Systems: A Framework for Analysis and Design. Abacus Press, Cambridge, MA, USA and Tunbridge Wells, Kent, UK.

[28] Lee, C. (1990) Fuzzy Logic in Control Systems: Fuzzy Logic Controller. I. IEEE Transactions on Systems, Man, and Cybernetics, 20, 404-418. https://doi.org/10.1109/21.52551

[29] Mamdani, E.H. (1977) Application of Fuzzy Logic to Approximate Reasoning Using Linguistic Syntheses. IEEE Transactions on Computers, C-26, 1182-1191. https://doi.org/10.1109/TC.1977.1674779

[30] Mas, M., Monserrat, M., Torrens, J. and Trillas, E. (2007) A Survey on Fuzzy Impli- 
cation Functions. IEEE Transactions on Fuzzy Systems, 15, 1107-1121. https://doi.org/10.1109/TFUZZ.2007.896304

[31] Mendel, J. (2005) References for Type-2 Fuzzy Sets and Fuzzy Logic Systems. http://sipi.usc.edu/ mendel/publications/T2\%20FS\%20\&\%20FLSs\%20Refs.pdf

[32] Mizumoto, M., Fukami, S. and Tanaka, K. (1979) Some Methods of Fuzzy Reasoning, In: Gupta, R. and Yager, R., Eds., Advances in Fuzzy Set Theory Applications, North-Holland, New York.

[33] Mizumoto, M. and Zimmermann, H.-J. (1982) Comparison of Fuzzy Reasoning Methods. Fuzzy Sets and Systems, 8, 253-283.

[34] Oh, K.-W. and Bandler, W. (1988) Properties of Fuzzy Implication Operators. Department of Computer Science, Florida State University, Tallahassee, FL, 24-33.

[35] Rescher, N. (1969) Many-Valued Logic. McGraw-Hill, New York.

[36] Serruier, M., Dubois, D., Prade, H. and Sudkamp, T. (2007) Learning Fuzzy Rules with Their Implication Operator. Data \& Knowledge Engineering, 60, 71-89.

[37] Stoll, R.R. (1979) Set Theory and Logic. Dover Publications, New York.

[38] Wenstøp, F. (1980) Quantitative Analysis with Linguistic Values. Fuzzy Sets and Systems, 4, 99-115.

[39] Willmott, R. (1978) Two Fuzzier Implication Operators in the Theory of Fuzzy Power Sets. In: Fuzzy Research Project, University of Essex, Colchester, UK, Department of Mathematics, FRP-2 Report.

[40] Willmott, R. (1980) Two Fuzzier Implication Operators in the Theory of Fuzzy Power Sets. Fuzzy Sets and Systems, 4, 31-36.

[41] Yager, R.R. (1999) On Global Requirements for Implication Operators in Fuzzy Modus Ponens. Fuzzy Sets and Systems, 106, 3-10.

[42] Zadeh, L.A. (1965) Fuzzy Sets. Information and Control, 8, 338-353.

[43] Zadeh, L.A. (1973) Outline of a New Approach to the Analysis of Complex Systems and Decision Processes. IEEE Transactions on Systems, Man, and Cybernetics, SMC-3, 28-44. https://doi.org/10.1109/TSMC.1973.5408575

[44] Zadeh, L.A. (1975) The Concept of a Linguistic Variable and Its Application to Approximate Reasoning. Information Sciences, 8, 43-80.

[45] Zadeh, L.A. (1988) Fuzzy Logic. IEEE Computer, 21, 83-93. https://doi.org/10.1109/2.53

[46] Zimmermann, H.-J. (1993) Fuzzy Set Theory and Its Applications. 2nd Edition, Kluwer, Boston. 


\section{Appendix}

\section{Criterion I}

Ant 1: If $x$ is $P$ then $y$ is $Q$

Ant 2: $x$ is $P$

Cons: $y$ is $Q$.

\section{Criterion II-1}

Ant 1: If $x$ is $P$ then $y$ is $Q$

Ant 2: $x$ is very $P$

Cons: $y$ is very $Q$.

\section{Criterion II-2}

Ant 1: If $x$ is $P$ then $y$ is $Q$

Ant 2: $x$ is very $P$

Cons: $y$ is $Q$.

\section{Criterion III}

Ant 1: If $x$ is $P$ then $y$ is $Q$

Ant 2: $x$ is more or less $P$

Cons: $y$ is more or less $Q$.

\section{Criterion IV-1}

Ant 1: If $x$ is $P$ then $y$ is $Q$

Ant 2: $x$ is not $P$

Cons: $y$ is unknown

\section{Criterion IV-2}

Ant 1: If $x$ is $P$ then $y$ is $Q$

Ant 2: $x$ is not $P$

Cons: $y$ is not $Q$.

Submit or recommend next manuscript to SCIRP and we will provide best service for you:

Accepting pre-submission inquiries through Email, Facebook, LinkedIn, Twitter, etc. A wide selection of journals (inclusive of 9 subjects, more than 200 journals)

Providing 24-hour high-quality service

User-friendly online submission system

Fair and swift peer-review system

Efficient typesetting and proofreading procedure

Display of the result of downloads and visits, as well as the number of cited articles Maximum dissemination of your research work

Submit your manuscript at: http://papersubmission.scirp.org/

Or contact jsea@scirp.org 